\title{
PRECISIÓN DIAGNÓSTICA DEL TERMÓMETRO DE DISTRÉS EN NEO- PLASIAS HEMATOLÓGICAS
}

\author{
DIAGNOSTIC ACCURACY OF DISTRESS THERMOMETER IN HEMATOLOGICAL \\ CANCER
}

\begin{abstract}
Yolanda Andreu Vaílo, Ma José Galdón Garrido, Paula Martínez López y Olga Martínez Arroyo
\end{abstract}

Universidad de Valencia

Resumen

Objetivo: El presente trabajo extiende la investigación previa sobre la validez y precisión diagnóstica del Termómetro de Distrés (DT) para detectar distrés psicosocial en pacientes con cáncer españoles. EI DT es un instrumento habitual de screening, al que, recientemente $y$ con el objetivo de mejorar su exactitud diagnóstica, se ha propuesto añadir otras herramientas, como el Termómetro de Impacto (IT).

Método: En este estudio analizamos la sintomatología emocional que apresa el DT y la exactitud diagnóstica que alcanza sólo o combinado con el IT mediante dos procedimientos diferentes. Un total de 81 pacientes adultos con cáncer hematológico completaron el DT, la Escala de Quejas Coloreada (CCS), el IT y el Inventario Breve de Síntomas-18 (BSI-18). La precisión diagnóstica del DT e IT se exploró a través de medidas globales -Área Bajo la Curva ROC (AUC)-, medidas de ocurrencia (Se y Sp), medidas de discriminación (PPV y NPV) e índices de utilidad clínica (UIs).

Resultado: Los resultados obtenidos fueron comparables a los hallados en estudios previos indicando que el DT es adecuado para la tarea de "screening", si bien su rendimiento en la "identificación de caso" es limitado. Además, los índices de precisión asociados al uso combinado del DT y el IT sólo mostraron diferencias menores respecto a los obtenidos por el DT.
Abstract

Objective: The present study extends the previous investigation about validity and diagnostic accuracy of the DT for detecting psychosocial distress among Spanish cancer patients. The Distress Thermometer (DT) is a common screening tool, but other methods such as Impact Thermometer (IT)- have recently been proposed with the aim of improving its diagnostic accuracy. In this paper, we investigated the emotional symptomatology captured by the DT, and the diagnostic accuracy of both the DT alone and combined with the IT, using two possible combination methods.

Methods: A sample of 81 adult patients with hematologic cancer completed the DT, the Colored Complaint Scale (CCS), the IT and the Brief Symptom Inventory-18 (BSI18). Several indexes were calculated to study the diagnostic accuracy: the area under the receiver operating characteristics (ROC) curve (AUC); measures of occurrence (Se y Sp), measures of discrimination (PPV and NPV) and clinical utility indexes (UIs).

Results: The results of the DT were comparable with those found in previous studies, indicating that the DT is adequate for 'screening', but has limited value for 'case finding'. Furthermore, the DT and the IT combined show minor differences in accuracy indexes compared with the DT alone.

\section{Correspondencia:}

Yolanda Andreu. Departamento de Personalidad, Evaluación y Tratamientos Psicológicos. Avda. Blasco Ibáñez, 13, 46010. Valencia (España).

E-mail. yolanda.andreu@uv.es 
Conclusiones: DT e IT se posicionan como herramientas útiles para su uso rutinario en la práctica clínica diaria, proporcionando información psicosocial relevante sobre el paciente al profesional sanitario sin interferir con su labor asistencial.

Palabras clave: distrés psicosocial, cáncer, screening, Termómetro de Distrés, BSI-18.
Conclusiones: DT and IT are useful tools for routine use in clinical practice providing psychosocial relevant patient information to healthcare professional without interfering in their care task.

Keywords: Psychosocial distress, cancer, screening, Distress Thermometer, BSI-18.

\section{INTRODUCCIÓN}

Los estudios sobre prevalencia del distrés en cáncer revelan que aproximadamente tres de cada diez pacientes oncológicos tiene niveles significativos de distrés a lo largo del proceso de la enfermedad ${ }^{(1-5)}$. Asimismo, un extenso meta-análisis reciente $^{(6)}$ muestra que los trastornos emocionales están presentes en un 30-40\% de los pacientes oncológicos. No obstante, no alcanzan a la mitad los pacientes con distrés emocional que son identificados y derivados a los servicios de asistencia psicosocial correspondientes ${ }^{(7,8)}$. Hecho éste que conlleva importantes consecuencias negativas ${ }^{(9)}$ en calidad de vida ${ }^{(10)}$, satisfacción con la atención médica ${ }^{(11)}$, e, incluso, puede repercutir en los gastos de asistencia sanitaria: mayor demanda de atención del equipo oncológico ${ }^{(12)}$, mayor uso de los servicios de atención primaria y urgencias hospitalarias ${ }^{(13)}$, mayor probabilidad de pruebas diagnósticas y tratamientos innecesarios $^{5}$ y peor adherencia a las recomendaciones médicas ${ }^{(14-15)}$.

En la última década se ha reivindicado considerar al distrés emocional como el "sexto signo vital" en el tratamiento del

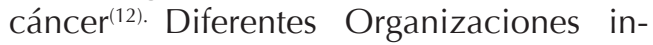
ternacionales -IPOS, NCCN, NICE- recomiendan incorporar el cribado rutinario y tratamiento del distrés como parte de la atención integral al paciente oncológico ${ }^{(16)}$. Disponer de una herramienta de cribado breve, precisa y aceptable es imprescin- dible para que un programa de screening resulte eficaz. En los últimos años se han adaptado escalas análogo-visuales (VAS) para evaluar dificultades emocionales. El ejemplo más conocido en el contexto del cáncer es el Termómetro de Distrés (Distress Thermometer, DT) ${ }^{(17)}$ que, con un solo ítem y una escala de 11 puntos $(0=a u-$ sencia de distrés y $10=$ distrés extremo) evalúa el grado de malestar emocional experimentado por el sujeto durante los últimos siete días. El DT es el instrumento de screening que, junto con un listado estandarizado de problemas para identificar las causas del distrés, sugiere utilizar la NCCN en su Guía de práctica clínica para el manejo de distrés ${ }^{(9)}$.

El DT ha sido validado y adaptado a diferentes lenguas, en pacientes con características médicas diferentes y utilizando básicamente el HADS y el BSI-18 como medidas criterio ${ }^{(17-20)}$. Los resultados revelan que el DT es una herramienta útil para la exclusión de casos no clínicos -objetivo prioritario de un instrumento de screening-, pero pobre para confirmar un posible caso de distrés moderado o severo -identificación de caso--21-22).

Son varias las propuestas que, sin sacrificar su brevedad y sensibilidad, pretenden mejorar la especificidad del DT. Algunas tratan de captar mejor el constructo de distrés psicosocial, incorporando otros termómetros emocionales. Así, argumentando la dificultad de encontrar una correspondencia exacta de la palabra "distrés" en 
poblaciones de habla no inglesa, se ha sugerido $^{(23)}$ el uso añadido de un Termómetro Emocional (Mood Thermometer, MT), que indague sobre el nivel de depresión que presenta el paciente. Los autores encuentran una ligera mejoría asociada a la inclusión del MT $(\Delta 4 \%$ especificidad y $\Delta 8 \%$ sensibilidad). Otra propuesta sugiere añadir información más específica sobre el estado de ánimo (ansiedad, depresión e ira) y sobre la necesidad de ayuda profesional, generando una herramienta compuesta por 5 termómetros emocionales (Emotion Thermometers Tool, ET5) (24). Una combinación específica del DT con los termómetros de depresión, ira y necesidad de ayuda mejoró los resultados del DT ( $\Delta 14 \%$ especificidad) ${ }^{(25)}$. Las propuestas de otros autores sugieren incluir información clínica relevante. El Barómetro de Distrés (DB) ${ }^{(26)}$ combina el DT con la Escala de Quejas Coloreada para cuantificar las diferentes fuentes de distrés. Con respecto al DT, el DB incrementa la especificidad $(11 \%)$, aunque disminuye ligeramente la sensibilidad (4\%). Otra sugerencia propone añadir un Termómetro de Impacto -IT- que recoja un componente clave de los criterios diagnósticos para los trastornos psiquiátricos: la interferencia del distrés experimentado en la vida cotidiana ${ }^{(27)}$. La combinación del DT $(\geq 4)$ y el IT $(\geq 3)$ parecen mantener la sensibilidad y mejorar significativamente la especificidad (23\%). También se ha explorado la posibilidad de incorporar la información proporcionada por el IT al DT, sumando las puntuaciones para obtener una única puntuación total ${ }^{(28)}$. No obstante, la suma (DIT $\geq 7$ ) no mejoró la precisión diagnóstica y la combinación de las puntuaciones (DT $\geq 3$ e IT $\geq 5$ ) mejoró ligeramente la especificidad (5\%) pero también disminuyó algo la sensibilidad (4\%).

Resultados previos en población oncológica española han mostrado que el DT $(\geq 5)$ presenta alta sensibilidad (90\%), aunque moderada especificidad $(64 \%)^{(29)}$ y que la combinación del DT y el IT (mediante suma o a partir de los puntos de corte establecidos) no permite alcanzar una precisión diagnóstica significativamente superior a la obtenida con el uso aislado del $\mathrm{DT}^{(30)}$. Estos trabajos han utilizado muestras oncológicas amplias y heterogéneas que abarcan diferentes localizaciones, tratamientos, fases de la enfermedad, etc. Sin embargo, existe un importante subgrupo de neoplasias que no se encuentra representado como tal en dichas muestras: las neoplasias hematológicas que representan el 5-6\% de todos los tumores humanos y comprenden un grupo heterogéneo entre los que se incluyen leucemias, linfomas y mielomas múltiples. Junto al aumento en prevalencia e incidencia que marca la creciente importancia de este subgrupo ${ }^{(31)}$, presenta además un perfil particular en cuanto al proceso asistencial (frecuencia de transfusiones, hospitalización para tratamiento quimioterapéutico, trasplantes de médula ósea, etc.) que requiere atención particular. El presente trabajo pretende extender la investigación previa sobre la utilidad y precisión diagnóstica del DT, así como sus posibilidades de mejora -incorporando el IT-, en un grupo de pacientes españoles adultos con neoplasias hematológicas.

\section{MÉTODO}

\section{Participantes}

La muestra esta compuesta por 81 pacientes. Alrededor del $60 \%$ tiene entre 40 y 60 años (Media=51 años, DT=10.7) y está laboralmente activo antes del ingreso hospitalario. Un 53\% son varones, un $80 \%$ tiene pareja y un $96 \%$ cursó al menos estudios primarios. Dos tercios tienen leucemia, un $16 \%$ linfoma y un $15 \%$ mieloma múltiple o síndrome mielodisplásico. Todos los pacientes están ingresados en el hospital debido al propio tratamiento $(90 \%)$ o a complicaciones médicas $(10 \%)$ (Ver tabla 1). 
Tabla 1. Estadísticos descriptivos de las variables sociodemográficas y médicas $(\mathbf{N}=\mathbf{8 1})$

\begin{tabular}{|c|c|c|c|c|c|}
\hline Variables sociodemográficas & $\mathbf{N}$ & $\%$ & & $\mathbf{N}$ & $\%$ \\
\hline Edad, Media (DT) & 51,2 & 10,66 & & & \\
\hline Sexo & & & tado civil & & \\
\hline Hombres & 43 & 53,1 & Casado/vive en pareja & 66 & 81,5 \\
\hline Mujeres & 38 & 46,9 & Soltero/viudo/separado/divorciado & 15 & 18,5 \\
\hline Nivel educativo & & & ctividad laboral & & \\
\hline Sin estudios & 3 & 3,7 & Tareas del hogar & 5 & 6,2 \\
\hline Estudios primarios & 43 & 53,1 & $\begin{array}{l}\text { Trabajo fuera de casa/laboralmente } \\
\text { activo }\end{array}$ & 51 & 63,0 \\
\hline Estudios secundarios & 19 & 23,5 & Retirado/de baja laboral & 15 & 18,5 \\
\hline Estudios superiores & 16 & 19,8 & Desempleado & 10 & 12,3 \\
\hline \multicolumn{6}{|l|}{ Variables médicas } \\
\hline Subtipo neoplasia hematológica & & & otivos de ingreso & & \\
\hline Linfoma No Hodgkin & 8 & 9,9 & Quimioterapia ordinaria & 26 & 32,9 \\
\hline Linfoma Hodgkin & 5 & 6,2 & Trasplante & 31 & 39,2 \\
\hline Mieloma múltiple & 9 & 11,1 & Tratamiento específico & 12 & 15,2 \\
\hline Leucemia linfoide & 16 & 19,8 & Complicaciones médicas & 10 & 12,7 \\
\hline Leucemia mieloide & 40 & 49,4 & & & \\
\hline Síndrome mielodisplásico & 3 & 3,7 & & & \\
\hline
\end{tabular}

Instrumentos

- Una hoja de datos recogió los datos sociodemográficos y médicos.

- "Termómetro de distrés" (Distress Thermometer, DT ${ }^{(17)}$. El DT emplea una escala visual de 11 puntos $(0$, "ningún malestar emocional" y 10 , "malestar emocional extremo") para evaluar el malestar emocional experimentado durante la última semana. Se utilizó la versión española del DT ${ }^{(29)}$.

- "Termómetro de impacto" (Impact Thermometer, IT) ${ }^{(27)}$. El IT emplea una escala visual de 11 puntos $(0$, "ausencia de interferencia" y 10, "interferencia extrema") para determinar la interferencia en la vida cotidiana del malestar emocional. Se utilizó la versión española del IT ${ }^{(30)}$.

- "Combinación de los termómetros de distrés (DT) e impacto (IT)". Se utilizaron tanto puntuaciones pareadas -establecimiento del punto de corte para cada instrumento y determinación de caso positivo sólo cuando se superan los dos puntos de corte establecidos ${ }^{(27)}$ - como puntuaciones sumadas -suma de las puntuaciones en ambos instrumentos y establecimiento de un único punto de corte $^{(28)}$-. Los puntos de corte seleccionados para el DT e IT fueron $\geq 5$ y para el DIT $\geq 9^{(30)}$.

- "Escala coloreada" (Colored Complaint Scale, $C C S)^{(26)}$. Identifica y cuantifica las fuentes de estrés percibido a través de 9 ítems que se responden según una escala coloreada con 5 opciones de respuesta (0, "ninguna molestia" y 4, "mucha molestia". La existencia de un problema se concreta dicotomizando las alternativas de respuesta: ausencia $(0,1)$ y presencia $(2,3$ y 4$)$.

- "Inventario breve de síntomas-18" (Brief Sympton Inventory-18, BSI$18)^{(32)}$. Autoinforme compuesto por 18 ítems (escala Likert 5 puntos) 
que evalúa el malestar emocional. El instrumento ofrece puntuaciones en somatización, depresión y ansiedad y una puntuación total (Global Severity Index; GSI) en malestar emocional. Se consideran casos con malestar clínicamente significativo a aquellos con una puntuación $\mathrm{T} \geq 63$ en la puntuación total $\mathrm{O}$, al menos, en dos de las subescalas ${ }^{(32)}$. EI BSI-18 ha mostrado propiedades psicométricas satisfactorias en población oncológica española ${ }^{(33)}$. Su consistencia interna en la muestra presente fue satisfactoria $\left(\alpha_{\mathrm{GSI}}=0,85\right.$, $\left.\alpha_{D}=0,65, \alpha_{A}=0,76, \alpha_{S}=0,63\right)$.

\section{Procedimiento}

Este estudio fue aprobado por el comité de investigación del Hospital Universitario La Fe de Valencia. La muestra inicial la forman 100 pacientes consecutivos ingresados en el Servicio de Hematología del Hospital Universitario la Fe de Valencia entre febrero de 2013 y febrero de 2014. Los criterios de elección fueron: diagnóstico de neoplasia hematológica, no encontrarse en situación de aislamiento y edad $\geq 18$. Los pacientes interesados en participar en el estudio firmaron el consentimiento informado. Dado que un 19\% rechazó participar, la muestra final la componen 81 sujetos.

\section{Análisis de datos}

Los análisis fueron realizados utilizando el paquete estadístico SPSS para Windows (versión 19.0) y MedCalc (versión 12). El nivel de significación estadística utilizado fue $\leq 0,05$. Se calcularon estadísticos descriptivos para resumir los datos sociodemográficos, médicos y psicológicos y correlaciones bivariadas (prueba rho no paramétrica de Spearman, dado el no cumplimiento del supuesto de normali- dad) para explorar la validez convergente de los termómetros contemplados (DT, IT, DIT). Su precisión diagnóstica se exploró a través de varios índices: medidas globales -Área Bajo la Curva ROC (AUC) y diferencias estadísticas entre curvas ROC-; medidas de ocurrencia (Se y Sp) y medidas de discriminación (PPV y NPV)(34). Se calcularon, por último, los índices de utilidad clínica (UIs): el UI+ (Se x PPV) refleja la precisión en la detección de casos y el UI- (Sp x NPV) refleja la precisión en la exclusión de los no casos (screening) ${ }^{(35)}$.

\section{RESULTADOS}

\section{BSI-18, DT, IT, DIT y CS}

La media de las puntuaciones en el DT fue de 4,78 (DT=2,62; rango 0-10). El valor medio en GSI (BSI-18) fue 11,11 $(\mathrm{DT}=10,71$; rango $0-53)$ y el porcentaje de pacientes que cumplía el criterio para ser considerado "caso" fue 21\% (17/81). La correlación entre DT, IT, DIT (suma de las puntuaciones en DT e IT) y GSI (puntuación total delBSI-18) fue significativa en todos los casos; si bien, el DT $(r=0,58$; $p \leq 0,001)$ mostró la correlación más alta con dicha variable y el IT obtuvo $(r=0,34$; $p \leq 0,01)$ la más baja. Asimismo, los tres termómetros correlacionaron significativamente con las tres subescalas del BSI-18; aunque, de nuevo, las correlaciones más altas fueron las obtenidas por el DT $\left(r_{A}=\right.$ 0,$57 ; p \leq 0,001 ; r_{D}=0,54 ; p \leq 0,001 ; r_{S}=$ $0,38 ; p \leq 0,001)$ y las más bajas las correspondientes al IT $\left(r_{A}=0,25 ; p \leq 0,05\right.$; $\left.r_{D}=0,34 ; p \leq 0,01 ; r_{S}=0,28 ; p \leq 0,05\right)$ (Ver tabla 2).

Las principales fuentes de distrés psicológico presentes en la muestra fueron los problemas de tipo emocional (76\%) y físico (64\%), aunque también ocupaban un lugar importante las preocupaciones familiares (43\%). El dolor está presente en prácticamente uno de cada tres pacientes (31\%) y 
Tabla 2. Estadísticos descriptivos de DT, IT y DIT y correlaciones con el criterio

\begin{tabular}{lccccc}
\hline M (DT) & GSI (BSI-18) & $\begin{array}{c}\text { Ansiedad } \\
(\mathbf{B S I}-\mathbf{1 8})\end{array}$ & $\begin{array}{c}\text { Depresión } \\
(\mathbf{B S I}-\mathbf{1 8})\end{array}$ & $\begin{array}{c}\text { Somatización } \\
(\mathrm{BSI}-\mathbf{1 8})\end{array}$ \\
\hline DT & $4,78(2,62)$ &, $58^{* * *}$ &, $57^{* * *}$ &, $54^{* * *}$ &, $38^{* * *}$ \\
\hline IT & $3,55(3,67)$ &, $34^{* *}$ &, $25^{*}$ &, $34^{* *}$ &, $28^{*}$ \\
\hline DIT & $8,33(5,24)$ &, $53^{* * *}$ &, $46^{* * *}$ &, $51^{* * *}$ &, $38^{* * *}$ \\
\hline
\end{tabular}

Nota: ${ }^{* *} p \leq 0,001 ;{ }^{* *} p \leq 0,01 ;{ }^{*} p \leq 0,05$.

la tristeza fue el problema emocional más frecuente $(47 \%)$, aunque también el nerviosismo/ansiedad ocupa un lugar destacado $(38 \%)$. Los tres termómetros mostraron un patrón de correlaciones con las diferentes fuentes de distrés similar (aunque algo más bajo) al presentado por la puntuación total del BSI-18y presentaron correlaciones significativas con los problemas emocionales (Ver tabla 3). El DT mostró los valores de correlación más altos con nerviosismo/ ansiedad y tristeza $\left(r_{N / A}=0,50 ; p \leq 0,001\right.$; $\left.r_{T}=0,65 ; p \leq 0,001\right)$ mientras el IT mostró las correlaciones más bajas -de hecho, la asociación con nerviosismo/ansiedad fue sólo marginalmente significativa $\left(r_{N / A}=\right.$ 0,$\left.19 ; p=0,099 ; r_{T}=0,38 ; p \leq 0,001\right)$.
Precisión diagnóstica del DT e IT considerados tanto de forma aislada, como combinados

El valor del área bajo la curva (AUC) resultó ser satisfactorio $(\mathrm{A} \cup \mathrm{C}=0,82 ; 95 \% \mathrm{Cl}$ : $0,73-0,91$ ), indicando la buena capacidad clasificatoria del $\mathrm{DT}^{(34)}$. Asimismo, tanto las medidas de ocurrencia y discriminación, como los índices de utilidad clínica (sensibilidad $=0,94 ;$ especificidad $=0,48 ; \mathrm{VPP}$ $=0,42 ; \mathrm{PVN}=0,93 ; \mathrm{UI}+=, 39 ; \mathrm{UI}-=, 45)$ asociados al punto de corte $\geq 5$ del DT evidenciaron su mejor comportamiento para descartar posibles no casos de distrés (screening) que para detectar posibles casos de distrés clínicamente significativo.

\section{Tabla 3. Fuentes de distrés presentes en la muestra y correlaciones con BSI-18, DT, IT y DIT}

\begin{tabular}{|c|c|c|c|c|c|}
\hline & $\begin{array}{c}\text { Frecuencia } \\
\text { problema } \\
\% \text { (N) }\end{array}$ & $\begin{array}{c}\text { Correlación } \\
\text { GSI }\end{array}$ & $\begin{array}{c}\text { Correlación } \\
\text { DT }\end{array}$ & $\begin{array}{c}\text { Correlación } \\
\text { IT }\end{array}$ & $\begin{array}{c}\text { Correlación } \\
\text { DIT }\end{array}$ \\
\hline FÍSICOS & $64,2 \%(52)$ & $56^{* * *}$ & $46^{* * *}$ &, 17 & $35^{* *}$ \\
\hline Dolor & $30,9 \%(25)$ & $48^{* * *}$ & $36^{* *}$ & ,03 & ,20 \\
\hline Otros & $34,6 \%(28)$ &, $43^{* * *}$ & $39 * * *$ & $24^{*}$ & $37^{* * *}$ \\
\hline EMOCIONALES & $76,0 \%(62)$ &, $75^{* * *}$ & $60 * * *$ &, $37^{* * *}$ &, $56 * * *$ \\
\hline Nerviosismo/ansiedad & $38,3 \%(31)$ & $64^{* * *}$ &, $50 * * *$ &, 19 & $39 * * *$ \\
\hline Tristeza & $46,9 \%(38)$ & $60 * * *$ & $65 * * *$ & $38 * * *$ & $60 * * *$ \\
\hline Ira & $25,9 \%(21)$ & $58^{* * *}$ & $26^{*}$ & $39 * * *$ & $41^{* * *}$ \\
\hline COGNITIVOS (Memoria) & $16,0 \%(13)$ & $45^{* * *}$ & $29 *$ &, $36 * *$ & $40 * * *$ \\
\hline PREOCUPACIONES FAMILIARES & $43,2 \%(35)$ & $46^{* * *}$ &, 17 & $25^{*}$ & $26^{*}$ \\
\hline ESPIRITUALES (Sentido de la vida) & $18,5 \%(15)$ &, $60^{* * *}$ &, $32 * *$ &, 21 & $31^{* *}$ \\
\hline OTROS (Trabajo, Soledad) & $8,6 \%(7)$ & $24^{*}$ & 10 & 11 & 13 \\
\hline
\end{tabular}

Nota: Dado el referente similar, se han unido las categorías de nerviosismo y ansiedad. ${ }^{* * *} p \leq 0,001 ;{ }^{* *} p \leq 0,01$; ${ }^{*} p \leq 0,05$ 
Los valores del área bajo la curva fueron adecuados para el IT (AUC $=0.67 ; 95 \% \mathrm{Cl}$ : $0,53-0,81)$ y el DIT (AUC $=0,77,95 \% \mathrm{Cl}$ : 0,64-0.88). No obstante, el IT obtuvo valores AUC (dif) significativamente inferiores a los del DT $($ dif $=0,152 ; p=0,050)$ y DIT (dif $=0,097 ; p=0,001)$; mientras que las diferencias entre DT y DIT no fueron significativas (dif $=0,055 ; p=0,309)$. La figura 1 muestra las curvas ROC para el DT, IT and DIT.

Los valores de sensibilidad, especificidad, VPP, VPN e IUs para cada una de las alternativas de medida se presentan en la tabla 4. Los valores más bajos fueron los obtenidos por el IT y ninguna de las dos combinaciones del DT e IT mostró resultados significativamente mejores que los obtenidos por el DT: ambas mejoraron la especificidad pero redujeron sustancialmente la sensibilidad. No obstante, la caída de los valores se mantiene en niveles adecuados cuando la combinación de las puntuaciones en DT e IT se realiza a través de la suma; si bien alcanza niveles altamente insatisfactorios cuando se combinan a partir de los puntos de corte en cada instrumento. El DT mostró el mejor UI positivo, mientras que las combinaciones del DT e IT mostraron mejores UIs negativos (Ver tabla 4).

Figura 1. Curvas ROC: Termómetro de Distrés (DT); Térmómetro de Impacto (IT); Termómetro DIT

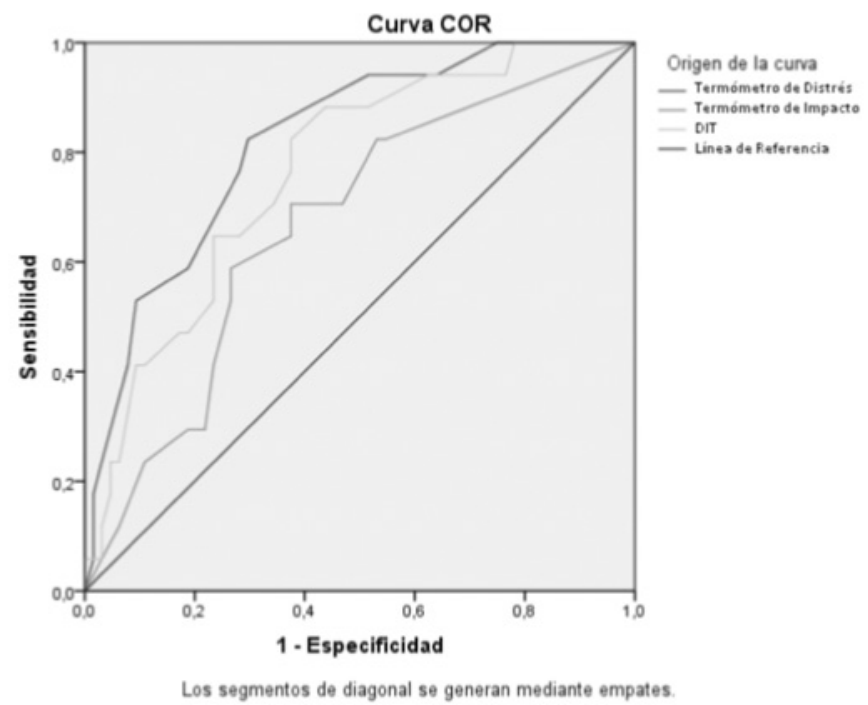

Tabla 4. Sensibilidad, especificidad, valor predictivo positivo, valor predictivo negativo e índices de utilidad de cada una de las medidas ultracortas

\begin{tabular}{ccccccc}
\hline & Se & Es & VPP & VPN & UI+ & UI- \\
\hline $\mathbf{D T} \geq \mathbf{5}$ &, 94 &, 48 &, 42 &, 93 &, 39 &, 45 \\
\hline $\mathbf{I T} \geq \mathbf{5}$ &, 64 &, 63 &, 37 &, 87 &, 24 &, 55 \\
\hline $\mathbf{D I T} \geq \mathbf{9}$ &, 82 &, 62 &, 35 &, 90 &, 29 &, 56 \\
\hline $\mathbf{D} \mathbf{T} \geq \mathbf{5} \mathbf{I T} \geq \mathbf{5}$ &, 53 &, 91 &, 60 &, 88 &, 32 &, 80 \\
\hline
\end{tabular}

Nota: $S e=$ sensibilidad, $E S=$ especificidad, $V P P=$ valor predictivo positivo, $V P N=$ valor predictivo negativo, UI+= indice de utilidad positivo, UI-= índice de utilidad negativo. 


\section{DISCUSIÓN / CONCLUSIONES}

El objetivo principal del estudio fue extender los resultados previos respecto al comportamiento del DT sólo o en combinación con el IT, como herramienta de cribado del distrés en pacientes con cáncer. Basándonos en las propuestas existentes ${ }^{(27-28)}$, combinamos el IT con el DT mediante suma o puntos de corte por separado.

Nuestros resultados confirman, en una muestra de pacientes adultos hospitalizados con neoplasias hematológicas, los datos previamente obtenidos ${ }^{(30)}$ respecto al buen comportamiento del DT como instrumento de cribado del distrés. Varios datos avalan su validez convergente. El DT correlacionó significativamente con la puntuación total -GSI- y con cada una de las subescalas -ansiedad, depresión, somatización- del BSI-18. En su asociación con las diferentes fuentes de distrés contempladas, el DT mostró un patrón similar -aunque con valores inferiores- al presentado por el BSI-18. Además, el DT mostró la correlación más alta con los problemas de origen emocional y se asoció con cada uno de los problemas emocionales concretos evaluados: "nerviosismo/ansiedad", "tristeza" y, en menor grado, "ira". Así y contrariamente a los resultados obtenidos por otros autores ${ }^{(26)}$, nuestros datos respaldan la validez del DT en cuanto medida del rango de respuestas emocionales que engloba el término distrés.

Nuestros resultados respaldan, asimismo, la precisión diagnóstica del DT ( $\mathrm{U} \cup \mathrm{C}=0,82)$ como herramienta de cribado. El DT ( $\geq 5)$ detectó un $94 \%$ de los casos positivos (Se) y un $48 \%$ de los casos negativos (Sp). Estos resultados, similares a los previamente encontrados en pacientes oncológicos españoles ${ }^{(1)}$, confirman el adecuado comportamiento del DT para descartar los no casos (screening) en pacientes con neoplasias hematológicas. No obstante, los datos respecto a sus niveles de especificidad, VPP e IU+ indican que necesita ser mejorado en lo que a "identificación de caso" se refiere ${ }^{(18-19)}$.

La combinación, mediante suma, del DT con el IT (DIT $\geq 9)$ mostró una validez convergente similar a la del DT. EI DIT obtuvo correlaciones significativas con la puntuación total y con cada una de las subescalas del BSI-18 y mostró una asociación significativa con las diferentes fuentes de distrés y, en particular, con los problemas emocionales. La exactitud diagnóstica, aunque algo más baja ( $\mathrm{A} \cup \mathrm{C}=0,77$ ), no fue significativamente diferente de la correspondiente al DT. Frente al DT, el DIT mostró una mayor especificidad (62\% vs. $48 \%$ ), aunque disminuyó ligeramente la sensibilidad ( $82 \%$ vs. 94\%). Los índices de utilidad del DIT recogen esta variación: el UI- aumentó (56\% vs. 45\%), mientras el UI+ disminuyó (29\% vs. $39 \%$ ). Los resultados mostraron un patrón bastante similar, aunque más polarizado, cuando DT e IT se combinaron según los puntos de corte ( $\geq 5$ en ambos): la especificidad aumentó sustancialmente con respecto al DT $(91 \%$ vs. $48 \%)$, pero la sensibilidad experimentó una drástica caída $(53 \%$ vs. 94\%), situándola en niveles inaceptables para una herramienta de cribado. Así pues, en contraste con los estudios previos que respaldan el uso combinado del DT y el $I T^{(27,28)}$ y en línea con los datos previamente obtenidos $^{(29)}$, nuestros resultados muestran que la inclusión del IT no mejora de manera sustantiva la precisión del DT como herramienta de cribado del distrés.

En suma, el DT constituye un procedimiento útil y preciso que, a la vez, puede resultar ágil y simple para cribar el distrés en pacientes con cáncer y, en su caso, realizar la pertinente derivación para evaluación psicológica. Hay dos argumentos, sin embargo, que pueden hacer que la combinación del IT con el DT constituya la alternativa de elección para el profesional 
de la salud. Nos referimos a la dificultad mínima que en tiempo y manejo conlleva la inclusión del IT y a la relevancia de la información que recoge este termómetro: la interferencia del distrés en la vida cotidiana. En caso de que sea ésta la opción escogida, conviene recordar que los datos respecto al uso combinado del DT y el IT en pacientes adultos hospitalizados con neoplasias hematológicas, respaldan el mejor funcionamiento de la combinación de las puntuaciones en ambos termómetros utilizando la suma(28).

El tamaño de la muestra estudiada y, en consecuencia, el pequeño número de sujetos identificados como pacientes con distrés clínicamente significativo (21\%) constituye una de las limitaciones del presente trabajo. No obstante, los resultados fueron establecidos a partir de medidas estándar de ocurrencia y discriminación que no están afectadas por la prevalencia de la condición de interés ${ }^{(34)}$. Medidas a las que incorporamos, además, otros indicadores relevantes de precisión diagnóstica como los índices de utilidad clínica, no considerados habitualmente en la investigación previa. La utilización de un instrumento de auto-informe -BSI-18- como criterio puede ser considerada, asimismo, otra limitación del presente trabajo. Aunque hubiera sido deseable el empleo, en su lugar, de una entrevista diagnóstica, un reciente metaanálisis ${ }^{(6)}$ ha mostrado que la prevalencia de cualquier trastorno emocional evaluado a través de auto-informe es similar a la encontrada cuando se utiliza una entrevista. Cabe señalar, por último, que un aspecto relevante que debería abordar la investigación sobre el uso de herramientas ultracortas en nuestro país es el análisis de otras características deseables en cualquier instrumento de screening como, por ejemplo, su aceptabilidad por parte de los profesionales de la salud y de los pacientes. El cribado rutinario del distrés sólo será viable si las personas implicadas consideran que las herramientas de screening resultan útiles y satisfactorias en la práctica clínica habitual.

\section{REFERENCIAS BIBLIOGRÁFICAS}

1. Andreu $Y$, Galdón MJ, Durá E, Martínez P, Pérez S,Murgui, S.A longitudinal study of psychosocial distress in breast cancer: Prevalence and risk factors. Psychol Health 2012; 27:72-87. Doi: 10.1080/08870446.2010.542814.

2. Carlson L, Angen M, Cullum J, Goodey E, Koopmans J, LamontL, et al. High levels of untreated distress and fatigue in cancer patients. Br J Cancer 2004; 90:2297-304. Doi:10.1038/sj.bjc.6601887

3. Grassi L, Travado L, Gil F, Sabato S, Rossi E, the SEPOS Group. Psychosocial morbidity and its correlates in cancer patients of the Mediterranean area: Findings from the Southern European Psycho-Oncology Study. J Affect Disord 2004; 83:243-8. Doi:10.1016/j.jad.2004.07.004

4. Hewitt M, Herdman R, Holland J. Meeting psychosocial needs of women with breast cancer. Washington, DC: The National Academic Press; 2004.

5. Zabora J, BrintzenhofeSzoc K, Curbow B, Hooker C, Piantadosi S. The prevalence of psychosocial distress by cancer site. Psychooncology 2001； 10:19-28. Doi:10.1002/1099$1611(200101 / 02) 10: 1<19::$ AID PON501>3.0.CO;2-6

6. Mitchell A, Chan M, Bhatti H, Halton M, Grassi L, Johansen C, et al. Prevalence of depression, anxiety, and adjustment disorder in oncological, hematological and palliative care settings: A meta-analysis of 94 interview-based studies. Lancet 2011;12:160-74. Doi. 10.1016/S14702045(11)70002-X

7. Kadan-Lottick NS, Vanderwerker LC, Block SD, Zhang B, Prigerson HG. Psychiatric disorders and mental health service use in patients with advanced cancer: A report 
from the coping with cancer study. Cancer 2005; 104: 2872-81. Doi.10.1002/ cncr.21532

8. Sharpe M, Strong V, Allen K, Rush R, Postma K, Tulloh A, et al. Major depression in outpatients attending a regional cancer center: Screening and unmet treatment needs. Br J Cancer 2004;90:314-20. Doi: 10.1038/sj.bjc.6601578

9. National Comprehensive Cancer Network [NCCN] (2014). Clinical practice guidelines in oncology. [Consultado el 18 de diciembre de 2014]. Disponible en: http:// www.nccn.org.

10. Shim E, Mehnert A, Koyama A, ChoS, Inui $H$, Paik $N$, et al. Health-related quality of life in breast cancer: Cross-cultural survey of Germany, Japanese, and South Korean patients. Breast Cancer Res Treat 2006;99:34150. Doi: 10.1007/s10549-006-9216-x

11. Von Essen L, Larsson G, Oberg K, Sjoden P. Satisfaction with care: Associations with health-related quality of life and psychosocial function among Swedish patients with endocrine gastrointestinal tumors. Eur J Cancer Care 2002;11:91-9. Doi. 10.1046/j.1365-2354.2002.00293.x

12. Bultz B, Holland J. Emotional Distress in patients with cancer: The sixth vital sign. Community Oncol 2006; 3:311-4.

13. Carlson L, Bultz B. Efficacy and medical cost offset of psychosocial interventions in cancer care: Making the case for economic analyses. Psychooncology 2004;13:83749. Doi:10.1002/pon.832

14. Kennard BD, Steward SM, Olvera R, Bawdon RE, OhAilin A, et al. Non-adherence in adolescent oncology patients: Preliminary data on psychological risk factors and relationships to outcome. J Clin Psychol Med Settings 2004;11:30-9.

15. DiMatteo M, Lepper $H$, Croghan T. Depression is a risk factor for noncompliance with medical treatment: Meta-analysis of the effects of anxiety and depression on patient adherence. Arch Intern Med 2000;160:2101-7.
16. Carlson LE, Waller A, Mitchell AJ. Screening for distress and unmet needs in patients with cancer: Review and recommendations. J Clin Oncol 2012; 30:11670. Doi: 10.1200/JCO.2011.39.5509

17. Roth AJ, Kornblith AB, Batel-Copel L, Peabody E, Scher HI, Holland JC. Rapid screening for psychological distress in men with prostate carcinoma: A pilot study. Cancer 1998;82:1904-8. Doi:10.1002/(SICI)1097$0142(19980515) 82: 10<1904:$ :AIDCNCR13>3.0.CO;2-X

18. Mitchell AJ. Pooled results from 38 analyses of the accuracy of distress thermometer and other ultra-short methods of detecting cancer-related mood disorders. J Clin Oncol 2007;25:4670-81. Doi: 10.1200/ JCO.2006.10.0438

19. Mitchell AJ. Short screening tools for cancer-related distress: A review and diagnostic validity meta-analysis. J Natl Compr Canc Netw 2010a; 8:487-94.

20. Vodermaier A, Linden W, Siu C. Screening for Emotional Distress in cancer patients: A systematic review of assessment instruments. J Natl Cancer Inst 2009;21:146488. Doi:10.1093/jnci/djp336

21. Donovan KA, Grassi L, McGinty HL, Jacobsen PB. Validation of the Distress Thermometer worldwide: State of the science. Psychooncology 2014;23:241-50. Doi:10.1002/pon.3430

22. Ma X, Zhang J, Zhong W, Shu C, Wang F, Wen J, et al. The diagnostic role of a short screening tool-the distress thermometer: A meta-analysis. Support Care Cancer 2014; 22:1741-55. Doi:10.1007/s00520-0142143-1.

23. Gil F, Grassi L, Travado L, Tormamichel M, González JM. Use of distress and depression thermometers to measure psychosocial morbidity among southern European cancer patients. Support Care Cancer 2005;13:600-6. Doi. 10.1007/s00520005-0780-0.

24. Mitchell AJ, Baker-Glenn EA, Granger L, Symonds P. Can the Distress Thermome- 
ter be improved by additional mood domains? Part I. Initial validation of the Emotion Thermometers tool. Psychooncology 2010; 19:125-33. Doi: 10.1002/pon.1523.

25. Mitchell AJ, Baker-Glenn EA, Park B, Granger L, Symonds P. Can the Distress Thermometer be improved by additional mood domains? Part II. What is the optimal combination of emotion thermometers? Psychooncology 2010;19:134-40. Doi:10.1002/pon.1557.

26. Bauwens $S$, Baillon C, Distelmans W, Theuns P. The "Distress Barometer": validation of method of combining the Distress Thermometer with a rated complaint scale. Psychooncology 2009;5:534-42. Doi:10.1002/pon.1425

27. Akizuki N, Yamawaki S, Akechi T, NakanoT, Uchitomi Y. Development of an Impact Thermometer for use in combination with the Distress Thermometer as a brief screening tool for adjustment disorders and/ or major depression in cancer patients. J Pain Symptom Manage 2005;1:91-9. Doi: 10.1016/j.jpainsymman.2004.04.016

28. Baken D, Woolley C. Validation of the Distress Thermometer, Impact Thermometer and combinations of these in screening for distress. Psychooncology 2001;20:609-14. Doi: 10.1002/pon.1934

29. Martínez P, Galdón MJ, Andreu Y, Ibáñez E. The distress Thermometer in Spanish cancer patients: Convergent validity and diagnostic accuracy. Support Care Cancer 2013;11:3095-102.

30. Martínez, P, Andreu Y, Galdón, MJ, Ibáñez, E. Improving the Diagnostic Accuracy of the Distress Thermometer: A Potential Role for the Impact Thermometer. J Pain Symptom Manage 2015;50:124-9. Doi:10.1016/j.jpainsymman.2015.02.004

31. Ferlay J, Soerjomataram I, Ervik M, DikshitR, Eser S, Mathers C, et al. Cancer incidence and mortality worldwide: IARC GLOBOCAN, 1 (11). 2012. [Consultado el 14 de junio de 2014] Disponible en: http://globocan.iarc.fr.

32. Derogatis LR. Brief Symptom Inventory (BSI-18). Administration, scoring and procedures manual. Minneapolis, MN: NCS Pearson; 2000.

33. Galdón MJ, Durá E, Andreu Y, Ferrando M, Murgui S, Pérez S, Ibáñez E. Psychometric proprieties of the Brief Symptoms Inventory 18 (BSI-18) in a Spanish breast sample. J Psychosom Res 2008;65:533-9. Doi:10.1016/j.jpsychores.2008.05.009

34. Franco M, Vivo JM. Cuadernos de estadística: Análisis de curvas ROC. Principios básicos y aplicaciones. Madrid: La MuraIla S.A; 2007.

35. Mitchell AJ. Clinical Utility Index - A new method to calculate the clinical value of diagnostic \& screening tests: Proof of concept study. Psychooncology 2012,19 S2 (S1-S325). 